\title{
AN EVALUATION OF THE INTEGRAL OF THE PRODUCT OF THE ERROR FUNCTION AND THE NORMAL PROBABILITY DENSITY WITH APPLICATION TO THE BIVARIATE NORMAL INTEGRAL
}

\author{
HATEM A. FAYED AND AMIR F. ATIYA
}

\begin{abstract}
This paper derives the value of the integral of the product of the error function and the normal probability density as a series of the Hermite polynomial and the normalized incomplete Gamma function. This expression is beneficial, and can be used for evaluating the bivariate normal integral as a series expansion. This expansion is a good alternative to the well-known tetrachoric series, when the correlation coefficient, $\rho$, is large in absolute value.
\end{abstract}

\section{INTRODUCTION}

This paper considers evaluation of the integral of the form:

$$
I(a, b, x)=\int_{0}^{x} e^{-t^{2}} \int_{0}^{a t+b} e^{-s^{2}} d s d t=\frac{\sqrt{\pi}}{2} \int_{0}^{x} e^{-t^{2}} \operatorname{erf}(a t+b) d t .
$$

To our knowledge, this integral has no closed form or even a single series expansion. However, Nicholson (1943) studied a simpler function, $V$, given by

$$
V(h, q)=\frac{1}{2 \pi} \int_{0}^{h} e^{-u^{2} / 2} \int_{0}^{q u / h} e^{-s^{2} / 2} d s d u=\frac{1}{2 \sqrt{\pi}} \int_{0}^{h / \sqrt{2}} e^{-t^{2}} \operatorname{erf}\left(\frac{q t}{h}\right) d t .
$$

Nicholson tabulated the function for $h, q=0(0.1) 3$ and for $q=\infty$. He obtained the following double expansion for computing $V(h, q)$ when $q<h$,

$$
V(h, q)=\frac{1}{2 \pi} \sum_{n=0}^{\infty}\left(1-e^{-h^{2} / 2} \sum_{j=0}^{n} \frac{h^{2 j}}{j ! 2^{j}}\right) \frac{(-1)^{n}}{(2 n+1)}\left(\frac{q}{h}\right)^{2 n+1} .
$$

Note that it can also be written as a single expansion as

$$
V(h, q)=\frac{1}{2 \pi} \sum_{n=0}^{\infty} P\left(n+1, \frac{h^{2}}{2}\right) \frac{(-1)^{n}}{(2 n+1)}\left(\frac{q}{h}\right)^{2 n+1},
$$

Received by the editor October 5, 2011 and February 20, 2012.

2010 Mathematics Subject Classification. Primary 33B20, 33C45.

Key words and phrases. Error function, normal probability, Gamma function, Hermite polynomial, hypergeometric function, bivariate normal integral, tetrachoric series. 
where $P(n, x)$ is the normalized incomplete Gamma function given by (Abramowitz and Stegun, 1964, p. 260, 262)

$$
P(n, x)=\frac{1}{\Gamma(n)} \int_{0}^{x} t^{n-1} e^{-t} d t=1-e^{-x} \sum_{j=0}^{n-1} \frac{x^{j}}{j !} .
$$

To evaluate $V(h, q)$ for $q>h$, Nicholson used the identity,

$$
V(h, q)+V(q, h)=\frac{1}{2 \pi} \int_{0}^{h} e^{-u^{2} / 2} d u \int_{0}^{q} e^{-s^{2} / 2} d s .
$$

Briggs (2003) listed some known integrals involving the error function and the normal probability density such as

$$
\begin{gathered}
\int_{-\infty}^{\infty} e^{-(\alpha t+\beta)^{2}} \operatorname{erf}(a t+b) d t=\frac{\sqrt{\pi}}{\alpha} \operatorname{erf}\left[\frac{\alpha b-\beta a}{\sqrt{\alpha^{2}+a^{2}}}\right], \\
\int_{0}^{\infty} e^{-(\alpha t)^{2}} \operatorname{erf}(\beta t) d t=\frac{1}{\alpha \sqrt{\pi}} \arctan \left(\frac{\beta}{\alpha}\right) .
\end{gathered}
$$

An important usage of the integral (1.1) is in the evaluation of the bivariate normal integral which has several applications. In the statistics field, Pearson 1901, 1903) used the bivariate normal distribution for studying biometric data. Evans and Schwab (1995) and Wooldridge (2001) made use of it in designing the binary endogenous regressor probit model (BERP). The BERP model is often used for computing the average treatment effect of the controlled versus the treated groups, as well as in other applications. Segregation analysis of the multifactorial model also needs the evaluation of bivariate (or multivariate) normal probabilities. In the finance field, the bivariate normal integral is used in the valuation of several types of exotic options (Dash, 2004, p. 269), Martzoukos (2001); Vasicek (1998). It is also used in computing the correlation of financial derivatives, as well as in computing the covariance of defaults on two loans (Vasicek, 1998). Bivariate integrals are also widely used in the communications field (such as the computation of bit error probabilities); see the extensive review of communications applications in (Simon and Dovsalar, 1998). In these applications, it is required to evaluate the bivariate normal cumulative distribution function. So far there is no general closed form expression for the bivariate normal integral, however, the Owen-Donnelly algorithm (Donnelly, 1973) offers an accurate numerical evaluation and has been extensively used in the literature as a benchmark. Nevertheless, it is still helpful to have an explicit formula for analytical development especially for preliminary calculations, and for computerized use when a large number of evaluations are required and speed of computation is important.

\section{Evaluation of the Integral}

In this section, we derive the result of the integral (1.1) as an infinite series of the normalized incomplete Gamma function and Hermite polynomial. 
Using the series expansions of the exponential and error functions, we get

$$
I(a, b, x)=\int_{t=0}^{x} \sum_{q=0}^{\infty} \frac{(-1)^{q}}{q !} t^{2 q} \sum_{n=0}^{\infty} \frac{(-1)^{n}}{n !(2 n+1)}(a t+b)^{2 n+1} d t .
$$

Using the binomial expansion of $(a t+b)^{2 n+1}$ and integrating, we get

$$
I(a, b, x)=\sum_{q=0}^{\infty} \sum_{n=0}^{\infty} \sum_{m=0}^{2 n+1} \frac{(-1)^{n+q} 2 n ! a^{2 n-m+1} x^{2 n+2 q-m+2} b^{m}}{(2 n+2 q-m+2) q ! n ! m ! 2 n-m+1 !} .
$$

Let us divide the inner summation into two summations for even and odd values of $m$ as follows:

$$
\begin{aligned}
I(a, b, x) & =\sum_{q=0}^{\infty} \sum_{n=0}^{\infty} \sum_{m=0}^{n} \frac{(-1)^{n+q} \Gamma(2 n+1)}{q ! \Gamma(n+1)} \\
& \cdot\left\{\frac{a^{2 n-2 m+1} x^{2 n+2 q-2 m+2} b^{2 m}}{(2 n+2 q-2 m+2) \Gamma(2 m+1) \Gamma(2 n-2 m+2)}\right. \\
& \left.+\frac{a^{2 n-2 m} x^{2 n+2 q-2 m+1} b^{2 m+1}}{(2 n+2 q-2 m+1) \Gamma(2 m+2) \Gamma(2 n-2 m+1)}\right\} \\
& =I_{1}+I_{2} .
\end{aligned}
$$

$I_{1}$ can be written with the interior summation changed to start from $m=-\infty$ (this would not change the sum since the added terms are all zeros), then using the transformation: $u=n-m$ leads to

$$
I_{1}=\sum_{q=0}^{\infty} \sum_{n=0}^{\infty} \sum_{u=0}^{\infty} \frac{(-1)^{n+q} \Gamma(2 n+1) a^{2 u+1} x^{2 u+2 q+2} b^{2 n-2 u}}{q !(2 u+2 q+2) \Gamma(n+1) \Gamma(2 n-2 u+1) \Gamma(2 u+2)}
$$

By changing the order of summation and using the transformation: $v=n-u$, we get

$$
I_{1}=\sum_{u=0}^{\infty} \sum_{q=0}^{\infty} \sum_{v=0}^{\infty} \frac{(-1)^{u+v+q} \Gamma(2 u+2 v+1) a^{2 u+1} x^{2 u+2 q+2} b^{2 v}}{q !(2 u+2 q+2) \Gamma(u+v+1) \Gamma(2 v+1) \Gamma(2 u+2)},
$$

where the inner summation counter is changed to start from $v=0$ rather than $v=-u$ since for $v<0$ the terms vanish. Using the duplication formula of Gamma function (Abramowitz and Stegun, 1964, p. 256) and the series expansion of the normalized incomplete Gamma function (Abramowitz and Stegun, 1964, p. 260, 262):

$$
\begin{aligned}
\Gamma(2 x) & =\frac{2^{2 x-1}}{\sqrt{\pi}} \Gamma(x) \Gamma\left(x+\frac{1}{2}\right), \\
P(a, x) & =\frac{1}{\Gamma(a)} \sum_{q=0}^{\infty} \frac{(-1)^{q} x^{q+a}}{q !(q+a)},
\end{aligned}
$$


we get

$$
\begin{aligned}
I_{1} & =\sum_{u=0}^{\infty} \sum_{q=0}^{\infty} \sum_{v=0}^{\infty} \frac{(-1)^{u+v+q} 2^{2 u} \Gamma\left(u+v+\frac{1}{2}\right)}{(2 u+2 q+2) q ! \Gamma\left(v+\frac{1}{2}\right) \Gamma(v+1) \Gamma(2 u+2)} a^{2 u+1} x^{2 u+2 q+2} b^{2 v} \\
& =\frac{1}{4} \sum_{u=0}^{\infty} \frac{(-1)^{u} \Gamma\left(u+\frac{1}{2}\right) a^{2 u+1}}{\Gamma\left(u+\frac{3}{2}\right)} \sum_{q=0}^{\infty} \frac{(-1)^{q}\left(x^{2}\right)^{q+u+1}}{q ! \Gamma(u+1)(q+u+1)} \sum_{v=0}^{\infty} \frac{\left(u+\frac{1}{2}\right)_{v}\left(-b^{2}\right)^{v}}{\left(\frac{1}{2}\right)_{v} v !} \\
& =\frac{1}{2} \sum_{u=0}^{\infty} \frac{(-1)^{u} a^{2 u+1}}{(2 u+1)} P\left(u+1, x^{2}\right)_{1} F_{1}\left(u+\frac{1}{2}, \frac{1}{2} ;-b^{2}\right),
\end{aligned}
$$

where $(\alpha)_{v}=\alpha(\alpha+1) \cdots(\alpha+v-1),(\alpha)_{0}=1$ and ${ }_{1} F_{1}(\alpha, \beta ; x)$ is the confluent hypergeometric function defined by (Andrews, 1985, p. 298)

$$
{ }_{1} F_{1}(\alpha, \beta ; x)=\sum_{v=0}^{\infty} \frac{(\alpha)_{v}}{v !(\beta)_{v}} x^{v} .
$$

Following a similar procedure, $I_{2}$ can be expressed as

$$
\begin{aligned}
I_{2} & =\sum_{u=0}^{\infty} \sum_{q=0}^{\infty} \sum_{v=0}^{\infty} \frac{(-1)^{u+v+q} \Gamma(2 u+2 v+1)}{q !(2 u+2 q+1) \Gamma(u+v+1) \Gamma(2 v+2) \Gamma(2 u+1)} a^{2 u} x^{2 u+2 q+1} b^{2 v+1} \\
& =\frac{\operatorname{sign}(x)}{2} \sum_{u=0}^{\infty} \frac{(-1)^{u} a^{2 u} b}{\Gamma(u+1)} \sum_{q=0}^{\infty} \frac{(-1)^{q}\left(x^{2}\right)^{q+u+\frac{1}{2}}}{q !\left(q+u+\frac{1}{2}\right)} \sum_{v=0}^{\infty} \frac{\left(u+\frac{1}{2}\right)_{v}}{\left(\frac{3}{2}\right)_{v} v !}\left(-b^{2}\right)^{v} \\
& =\frac{\operatorname{sign}(x)}{2} \sum_{u=0}^{\infty} \frac{(-1)^{u} \Gamma\left(u+\frac{1}{2}\right) a^{2 u} b}{\Gamma(u+1)} P\left(u+\frac{1}{2}, x^{2}\right)_{1} F_{1}\left(u+\frac{1}{2}, \frac{3}{2} ;-b^{2}\right) .
\end{aligned}
$$

Therefore,

$$
\begin{aligned}
I(a, b, x) & =\frac{\sqrt{\pi}}{2} \int_{t=0}^{x} e^{-t^{2}} \operatorname{erf}(a t+b) d t=I_{1}+I_{2} \\
& =\frac{1}{2} \sum_{u=0}^{\infty}\left\{\frac{(-1)^{u} a^{2 u+1}}{(2 u+1)} P\left(u+1, x^{2}\right)_{1} F_{1}\left(u+\frac{1}{2}, \frac{1}{2} ;-b^{2}\right)\right. \\
& \left.+\operatorname{sign}(x) \frac{(-1)^{u} \Gamma\left(u+\frac{1}{2}\right) a^{2 u} b}{\Gamma(u+1)} P\left(u+\frac{1}{2}, x^{2}\right)_{1} F_{1}\left(u+\frac{1}{2}, \frac{3}{2} ;-b^{2}\right)\right\} .
\end{aligned}
$$

This formula can be expressed in terms of the Hermite polynomial and the normalized incomplete Gamma function using the following relations (Andrews, 1985, p. 309, 313):

$$
\begin{gathered}
\int_{0}^{\infty} t^{2 u} \cos (2 x t) e^{-t^{2}} d t=\frac{1}{2} \Gamma\left(u+\frac{1}{2}\right)_{1} F_{1}\left(u+\frac{1}{2}, \frac{1}{2} ;-x^{2}\right)=\sqrt{\pi} \frac{(-1)^{u}}{2^{2 u+1}} e^{-x^{2}} H_{2 u}(x), \\
\int_{0}^{\infty} t^{2 u+1} \sin (2 x t) e^{-t^{2}} d t=x \Gamma\left(u+\frac{3}{2}\right)_{1} F_{1}\left(u+\frac{3}{2}, \frac{3}{2} ;-x^{2}\right)=\sqrt{\pi} \frac{(-1)^{u}}{2^{2 u+2}} e^{-x^{2}} H_{2 u+1}(x),
\end{gathered}
$$

where $H_{j}(x)$ is the Hermite polynomial defined by

$$
H_{j}(x)=j ! \sum_{k=0}^{[j / 2]} \frac{(-1)^{k}}{k !(j-2 k) !}(2 x)^{j-2 k}
$$


and

$$
\begin{aligned}
{ }_{1} F_{1}\left(\frac{1}{2}, \frac{3}{2} ;-x^{2}\right) & =\frac{1}{x \sqrt{\pi}} \int_{0}^{\infty} \frac{\sin (2 x t)}{t} e^{-t^{2}} d t=\frac{\sqrt{\pi}}{x}\left[\frac{1}{2}-\Phi(-\sqrt{2} x)\right], \\
P\left(\frac{1}{2}, x^{2}\right) & =2 \Phi(\sqrt{2}|x|)-1, \\
\Phi(x) & =\frac{1}{\sqrt{2 \pi}} \int_{-\infty}^{x} \exp \left(-\frac{t^{2}}{2}\right) d t \text { (standard univariate CDF). }
\end{aligned}
$$

Therefore,

$$
\begin{aligned}
I(a, b, x) & =\frac{\sqrt{\pi}}{2} \int_{t=0}^{x} e^{-t^{2}} \operatorname{erf}(a t+b) d t \\
& =I_{0}+\frac{\sqrt{\pi}}{2} e^{-b^{2}} \sum_{u=0}^{\infty}\left\{\frac{(a / 2)^{2 u+1}}{\Gamma\left(u+\frac{3}{2}\right)} P\left(u+1, x^{2}\right) H_{2 u}(b)\right. \\
& \left.-\frac{\operatorname{sign}(x)(a / 2)^{2 u+2}}{\Gamma(u+2)} P\left(u+\frac{3}{2}, x^{2}\right) H_{2 u+1}(b)\right\}
\end{aligned}
$$

where

$$
I_{0}=\frac{\pi}{2} \operatorname{sign}(x)(2 \Phi(\sqrt{2}|x|)-1)\left(\frac{1}{2}-\Phi(-\sqrt{2} b)\right) .
$$

Using the fact that $P(x, \infty)=1$, we get

$$
\begin{aligned}
I(a, b, \infty) & =\frac{\sqrt{\pi}}{2} \int_{t=0}^{\infty} \exp \left(-t^{2}\right) \operatorname{erf}(a t+b) d t \\
& =\frac{\pi}{2}\left(\frac{1}{2}-\Phi(-\sqrt{2} b)\right) \\
& +\frac{\sqrt{\pi}}{2} e^{-b^{2}} \sum_{u=0}^{\infty}\left\{\frac{(a / 2)^{2 u+1}}{\Gamma\left(u+\frac{3}{2}\right)} H_{2 u}(b)-\frac{(a / 2)^{2 u+2}}{\Gamma(u+2)} H_{2 u+1}(b)\right\}
\end{aligned}
$$

Using (1.3), it can be written as

$$
I(a, b, \infty)=\frac{\pi}{4} \operatorname{erf}\left(\frac{b}{\sqrt{1+a^{2}}}\right)+\frac{\sqrt{\pi}}{2} \exp \left(-b^{2}\right) \sum_{u=0}^{\infty} \frac{(a / 2)^{2 u+1}}{\Gamma\left(u+\frac{3}{2}\right)} H_{2 u}(b) .
$$

\section{Convergence}

Let us examine the convergence of (2.1). By using the fact that $0 \leq P\left(., x^{2}\right) \leq$

1 and the upper bound of Hermite polynomial (Abramowitz and Stegun, 1964, p. 787)

$$
\begin{gathered}
\left|H_{2 n}(x)\right| \leq \exp \left(\frac{x^{2}}{2}\right) 2^{2 n} n !\left[2-\frac{1}{2^{2 n}}\left(\begin{array}{l}
2 n \\
n
\end{array}\right)\right], \\
\left|H_{2 n+1}(x)\right| \leq|x| \exp \left(\frac{x^{2}}{2}\right) \frac{2 n+2 !}{n+1 !}, \quad x \geq 0,
\end{gathered}
$$


we get

$$
\begin{aligned}
\left|I(a, b, x)-I_{0}\right| & \leq e^{-b^{2} / 2}\left[\sum_{u=0}^{\infty}\left(\frac{\sqrt{\pi} u !}{2 \Gamma\left(u+\frac{3}{2}\right)}-\frac{1}{2(2 u+1)}\right) a^{2 u+1}\right. \\
& \left.+\left(\frac{|b| \Gamma\left(u+\frac{3}{2}\right)}{2 \Gamma(u+2)}\right) a^{2 u+2}\right] \\
& \leq e^{-b^{2} / 2} \sum_{u=0}^{\infty}\left[\left(\frac{\sqrt{\pi}}{2}-\frac{1}{2(2 u+1)}\right) a^{2 u+1}+\left(\frac{|b|}{2}\right) a^{2 u+2}\right],
\end{aligned}
$$

which converges for $|a|<1$.

\section{Application: Bivariate normal integral}

4.1. Literature review. The standard bivariate normal distribution of the random variables $X_{1}, X_{2}$ is given by

$$
f_{X_{1}, X_{2}}\left(x_{1}, x_{2}\right)=\frac{1}{2 \pi \sqrt{|\Sigma|}} \exp \left\{-\frac{1}{2}\left[\begin{array}{ll}
x_{1} & x_{2}
\end{array}\right]^{T} \Sigma^{-1}\left[\begin{array}{l}
x_{1} \\
x_{2}
\end{array}\right]\right\},
$$

where

$$
\Sigma=\left[\begin{array}{ll}
1 & \rho \\
\rho & 1
\end{array}\right]
$$

is the covariance matrix and $\rho$ is the correlation coefficient. The standard cumulative distribution function $(\mathrm{CDF})$ is given by

$$
\Phi(h, k, \rho)=\int_{x_{1}=-\infty}^{h} \int_{x_{2}=-\infty}^{k} f_{X_{1}, X_{2}}\left(x_{1}, x_{2}\right) d x_{2} d x_{1}
$$

and the complementary integral studied by many researchers (Divgi, 1979; Drezner and Wesolowsky 1990; Pearson, 1901; Simon and Divsalar, 1998) is

$$
L(h, k, \rho)=\int_{x_{1}=h}^{\infty} \int_{x_{2}=k}^{\infty} f_{X_{1}, X_{2}}\left(x_{1}, x_{2}\right) d x_{2} d x_{1} .
$$

These functions satisfy the following important equations (Gai, 2002):

$$
\begin{gathered}
\Phi(h, k, \rho)=L(-h,-k, \rho), \\
L(h, k, \rho)=1-\Phi(h)-L(h,-k,-\rho) .
\end{gathered}
$$

Sheppard (1900) was perhaps the first statistician who considered the problem of evaluation of the bivariate probability integral using the $V$ function defined by (1.2). Later, Pearson (1901) obtained the following simple tetrachoric series expansion:

$$
\Phi(h, k, \rho)=\Phi(h) \Phi(k)+\frac{1}{2 \pi} \exp \left\{-\frac{\left(h^{2}+k^{2}\right)}{2}\right\} \sum_{j=0}^{\infty} \frac{2^{-j} \rho^{j+1}}{j+1 !} H_{j}\left(\frac{h}{\sqrt{2}}\right) H_{j}\left(\frac{k}{\sqrt{2}}\right) .
$$

However, this series converges too slowly for high values of $|\rho|$ and thus is not very satisfactory.

Owen (1956) expressed the bivariate normal CDF in terms of the T-function as

$$
\Phi(h, k, \rho)=\frac{1}{2} \Phi(h)-T\left(h, \frac{k-\rho h}{h \sqrt{1-\rho^{2}}}\right)+\frac{1}{2} \Phi(k)-T\left(k, \frac{h-\rho k}{k \sqrt{1-\rho^{2}}}\right)+\eta,
$$


where

$$
\begin{aligned}
\eta & =\left\{\begin{aligned}
0 & \text { if } h k>0 \text { or if } h k=0 \text { but } h+k \geq 0 \\
-\frac{1}{2} & \text { otherwise, }
\end{aligned}\right. \\
T(h, a) & =\frac{1}{2 \pi} \int_{0}^{a} \frac{1}{1+x^{2}} \exp \left\{-\frac{h^{2}}{2}\left(1+x^{2}\right)\right\} d x \\
& =\frac{\arctan a}{2 \pi}-\frac{1}{2 \pi} \sum_{j=0}^{\infty} \frac{(-1)^{j} a^{2 j+1}}{2 j+1}\left[1-\exp \left(-\frac{h^{2}}{2}\right) \sum_{i=0}^{j} \frac{h^{2 i}}{2^{i} i !}\right] .
\end{aligned}
$$

Note that the T-function can also be written as:

$$
T(h, a)=\frac{\arctan a}{2 \pi}-\frac{1}{2 \pi} \sum_{j=0}^{\infty} \frac{(-1)^{j} a^{2 j+1}}{2 j+1} P\left(j+1, \frac{h^{2}}{2}\right) .
$$

The series expansion of the T-function converges rapidly for small values of $a$ and $h$ but converges quite slowly when $h$ is large and $a$ is close to 1 . Donnelly (1973) directly coded Owen's method and formed the Owen-Donnelly algorithm, which has been widely used as it gives the values of the bivariate normal CDF to 15 digit accuracy.

Vasicek (1998) derived another infinite series expansion that converges as a series of powers of $\left(1-\rho^{2}\right)$ for $h k \neq 0$,

$$
\Phi(h, k, \rho)=\Phi\left(h, 0, \frac{(\rho h-k) \operatorname{sign}(h)}{\sqrt{h^{2}-2 \rho h k+k^{2}}}\right)+\Phi\left(k, 0, \frac{(\rho k-h) \operatorname{sign}(k)}{\sqrt{h^{2}-2 \rho h k+k^{2}}}\right)-\beta,
$$

where

$$
\begin{gathered}
\beta= \begin{cases}0 & \text { if } h k>0, \\
\frac{1}{2} & \text { if } h k<0,\end{cases} \\
\Phi\left(x, 0, \rho_{0}\right)=\left\{\begin{array}{l}
\min \left(\Phi(x), \frac{1}{2}\right)-Q \\
\max \left(\Phi(x)-\frac{1}{2}, 0\right)+Q \quad \text { if } \rho_{0}>0,
\end{array}\right. \\
Q=\sum_{k=0}^{\infty} \frac{(-1)^{k} 2^{-k}|x|^{2 k+1}}{k !(2 k+1)} \alpha_{k}, \\
\alpha_{k}=\frac{1}{2 \pi} \exp \left\{-\frac{x^{2}}{2\left(1-\rho_{0}^{2}\right)}\right\} \sum_{i=0}^{k} \frac{(-1)^{i}(2 i) !}{2^{i} i !|x|^{2 i+1}}\left(1-\rho_{0}^{2}\right)^{i+\frac{1}{2}}-\frac{1}{\sqrt{2 \pi}} \Phi\left(-\frac{|x|}{\sqrt{1-\rho_{0}^{2}}}\right) .
\end{gathered}
$$

This method represents a good alternative when $|\rho|$ is large, however, it converges very slowly when $\left|\rho_{0}\right|$ is small, i.e., when $\rho h$ is close to $k$ or $\rho k$ is close to $h$.

In the following section, we derive two expansions: the first converges as a series of powers of $\sqrt{(1-\rho) /(1+\rho)}$, which can be used for $\rho>0$, while the other converges as a series of powers of $\sqrt{(1+\rho) /(1-\rho)}$, which can be used for $\rho<0$. Consequently, these series are more satisfactory for large $|\rho|$. These series have simpler forms compared to Owen's method and converge fast compared to Pearson and Vasicek methods for large $|\rho|$ even when $\rho h$ is close to $k$ or $\rho k$ is close to $h$.

There are some other methods in the literature that are based on numerically approximating the integral. Among those methods, Divgi (1979) estimated the complementary integral in polar coordinates. Drezner (1978) provided different 
formulas based on a direct computation of the double integral using Gauss quadrature method. Terza and Welland (1991) compared several algorithms for evaluating the bivariate normal CDF and judged that Divgi's algorithm is the best. Drezner and Wesolowsky (1990) presented a simple method that achieves single precision accuracy with less computation time than Divgi's algorithm. Genz (2004) presented a modified form of the Dresner and Wesolowsky algorithm that is slightly more accurate for double precision.

4.2. A novel expansion. To derive our new expansions, we start with the complementary integral (4.1) and write it after rotation of axes in a way that removes the cross product term by diagonalizing the inverse of the covariance matrix as $\Sigma^{-1}=P \Lambda P^{T}$, where

$$
P=\left[\begin{array}{ll}
\frac{1}{\sqrt{2}} & \frac{1}{\sqrt{2}} \\
\frac{1}{\sqrt{2}} & \frac{-1}{\sqrt{2}}
\end{array}\right]=P^{T}, \quad \Lambda=\left[\begin{array}{ll}
\frac{1}{1+\rho} & 0 \\
0 & \frac{1}{1-\rho}
\end{array}\right] .
$$

Hence, by using the transformation

$$
\left[\begin{array}{l}
y_{1} \\
y_{2}
\end{array}\right]=P^{T}\left[\begin{array}{l}
x_{1} \\
x_{2}
\end{array}\right] \Rightarrow\left[\begin{array}{l}
x_{1} \\
x_{2}
\end{array}\right]=P\left[\begin{array}{l}
y_{1} \\
y_{2}
\end{array}\right]
$$

the complementary integral can be expressed in the case $\rho>0$ as follows:

$$
\begin{aligned}
L(h, k, \rho) & =\frac{1}{2 \pi \sqrt{1-\rho^{2}}}\left\{\int_{y_{2}=\frac{h-k}{\sqrt{2}}}^{\infty} \int_{y_{1}=y_{2}+\sqrt{2} k}^{\infty} \exp \left\{-\frac{y_{1}^{2}}{2(1+\rho)}-\frac{y_{2}^{2}}{2(1-\rho)}\right\} d y_{1} d y_{2}\right. \\
& \left.+\int_{-\infty}^{y_{2}=\frac{h-k}{\sqrt{2}}} \int_{y_{1}=-y_{2}+\sqrt{2} h}^{\infty} \exp \left\{-\frac{y_{1}^{2}}{2(1+\rho)}-\frac{y_{2}^{2}}{2(1-\rho)}\right\} d y_{1} d y_{2}\right\}
\end{aligned}
$$

By scaling the variables

$$
z_{1}=\frac{y_{1}}{\sqrt{2(1+\rho)}}, z_{2}=\frac{y_{2}}{\sqrt{2(1-\rho)}}
$$

the integral becomes

$$
\begin{aligned}
L(h, k, \rho) & =\frac{1}{\pi}\left\{\int_{z_{2}=z_{20}}^{\infty} \int_{z_{1}=a_{1} z_{2}+b_{1}}^{\infty} e^{-z_{1}^{2}-z_{2}^{2}} d z_{1} d z_{2}+\int_{-\infty}^{z_{2}=z_{20}} \int_{z_{1}=-a_{1} z_{2}+c_{1}}^{\infty} e^{-z_{1}^{2}-z_{2}^{2}} d z_{1} d z_{2}\right\} \\
& =\frac{1}{\pi}\left\{\int_{z_{2}=z_{20}}^{\infty} \int_{z_{1}=a_{1} z_{2}+b_{1}}^{\infty} e^{-z_{1}^{2}-z_{2}^{2}} d z_{1} d z_{2}+\int_{z_{2}=-z_{20}}^{\infty} \int_{z_{1}=a_{1} z_{2}+c_{1}}^{\infty} e^{-z_{1}^{2}-z_{2}^{2}} d z_{1} d z_{2}\right\} \\
& =\frac{1}{\pi}\{J+K\},
\end{aligned}
$$

where

$$
a_{1}=\sqrt{\frac{1-\rho}{1+\rho}}, b_{1}=\frac{k}{\sqrt{1+\rho}}, c_{1}=\frac{h}{\sqrt{1+\rho}}, z_{20}=\frac{h-k}{2 \sqrt{1-\rho}}
$$


Let us investigate $J$ first:

$$
\begin{aligned}
J & =\int_{z_{2}=z_{20}}^{\infty} \int_{z_{1}=a_{1} z_{2}+b_{1}}^{\infty} e^{-z_{1}^{2}-z_{2}^{2}} d z_{1} d z_{2} \\
& =\int_{z_{2}=z_{20}}^{\infty} \int_{z_{1}=0}^{\infty} e^{-z_{1}^{2}-z_{2}^{2}} d z_{1} d z_{2}-\int_{z_{2}=z_{20}}^{\infty} \int_{z_{1}=0}^{a_{1} z_{2}+b_{1}} e^{-z_{1}^{2}-z_{2}^{2}} d z_{1} d z_{2} \\
& =\frac{\pi}{4} \operatorname{erfc}\left(z_{20}\right)-\frac{\sqrt{\pi}}{2} \int_{z_{2}=z_{20}}^{\infty} e^{-z_{2}^{2}} \operatorname{erf}\left(a_{1} z_{2}+b_{1}\right) d z_{2} \\
& =\frac{\pi}{4} \operatorname{erfc}\left(z_{20}\right)-\frac{\sqrt{\pi}}{2} \int_{z_{2}=0}^{\infty} e^{-z_{2}^{2}} \operatorname{erf}\left(a_{1} z_{2}+b_{1}\right) d z_{2} \\
& +\frac{\sqrt{\pi}}{2} \int_{z_{2}=0}^{z_{20}} e^{-z_{2}^{2}} \operatorname{erf}\left(a_{1} z_{2}+b_{1}\right) d z_{2} \\
& =\frac{\pi}{4} \operatorname{erfc}\left(z_{20}\right)-I\left(a_{1}, b_{1}, \infty\right)+I\left(a_{1}, b_{1}, z_{20}\right) .
\end{aligned}
$$

Using (2.1) and (2.2), where we have $a_{1}<1$ for $\rho>0$, we get

$$
\begin{aligned}
J & =\frac{\pi}{4} \operatorname{erfc}\left(z_{20}\right)-\frac{\pi}{2}\left(\frac{1}{2}-\Phi\left(-\sqrt{2} b_{1}\right)\right)\left(1-\operatorname{sign}\left(z_{20}\right)\left(2 \Phi\left(\sqrt{2}\left|z_{20}\right|\right)-1\right)\right) \\
& -\frac{\sqrt{\pi}}{2} e^{-b_{1}^{2}} \sum_{u=0}^{\infty}\left\{\frac{1}{\Gamma\left(u+\frac{3}{2}\right)}\left(\frac{a_{1}}{2}\right)^{2 u+1}\left(1-P\left(u+1, z_{20}^{2}\right)\right) H_{2 u}\left(b_{1}\right)\right. \\
& \left.-\frac{1}{\Gamma(u+2)}\left(\frac{a_{1}}{2}\right)^{2 u+2}\left(1+\operatorname{sign}\left(z_{20}\right) P\left(u+\frac{3}{2}, z_{20}^{2}\right)\right) H_{2 u+1}\left(b_{1}\right)\right\}
\end{aligned}
$$

and by analogy,

$$
\begin{aligned}
K & =\frac{\pi}{4} \operatorname{erfc}\left(-z_{20}\right)-\frac{\pi}{2}\left(\frac{1}{2}-\Phi\left(-\sqrt{2} c_{1}\right)\right)\left(1+\operatorname{sign}\left(z_{20}\right)\left(2 \Phi\left(\sqrt{2}\left|z_{20}\right|\right)-1\right)\right) \\
& -\frac{\sqrt{\pi}}{2} e^{-c_{1}^{2}} \sum_{u=0}^{\infty}\left\{\frac{1}{\Gamma\left(u+\frac{3}{2}\right)}\left(\frac{a_{1}}{2}\right)^{2 u+1}\left(1-P\left(u+1, z_{20}^{2}\right)\right) H_{2 u}\left(c_{1}\right)\right. \\
& \left.-\frac{1}{\Gamma(u+2)}\left(\frac{a_{1}}{2}\right)^{2 u+2}\left(1-\operatorname{sign}\left(z_{20}\right) P\left(u+\frac{3}{2}, z_{20}^{2}\right)\right) H_{2 u+1}\left(c_{1}\right)\right\} .
\end{aligned}
$$

Hence using $\operatorname{erfc}\left(z_{20}\right)+\operatorname{erfc}\left(-z_{20}\right)=2$, we end up with the following formula for $\rho>0$ :

$$
\begin{aligned}
L(h, k, \rho) & =L_{10}-\sum_{u=0}^{\infty}\left[A_{u}\left(1-P\left(u+1, z_{20}^{2}\right)\right)\left(e^{-b_{1}^{2}} H_{2 u}\left(b_{1}\right)+e^{-c_{1}^{2}} H_{2 u}\left(c_{1}\right)\right)\right. \\
& -B_{u} e^{-b_{1}^{2}} H_{2 u+1}\left(b_{1}\right)\left(1-\operatorname{sign}\left(z_{20}\right) P\left(u+\frac{3}{2}, z_{20}^{2}\right)\right) \\
& \left.-B_{u} e^{-c_{1}^{2}} H_{2 u+1}\left(c_{1}\right)\left(1+\operatorname{sign}\left(z_{20}\right) P\left(u+\frac{3}{2}, z_{20}^{2}\right)\right)\right]
\end{aligned}
$$


where

$$
\begin{aligned}
A_{u} & =\frac{1}{2 \sqrt{\pi} \Gamma\left(u+\frac{3}{2}\right)}\left(\frac{a_{1}}{2}\right)^{2 u+1}, B_{u}=\frac{1}{2 \sqrt{\pi} \Gamma(u+2)}\left(\frac{a_{1}}{2}\right)^{2 u+2}, \\
L_{10} & =\frac{1}{2}-\frac{1}{2}\left(\frac{1}{2}-\Phi\left(-\sqrt{2} b_{1}\right)\right)\left(1-\operatorname{sign}\left(z_{20}\right)\left(2 \Phi\left(\sqrt{2}\left|z_{20}\right|\right)-1\right)\right) \\
& -\frac{1}{2}\left(\frac{1}{2}-\Phi\left(-\sqrt{2} c_{1}\right)\right)\left(1+\operatorname{sign}\left(z_{20}\right)\left(2 \Phi\left(\sqrt{2}\left|z_{20}\right|\right)-1\right)\right) .
\end{aligned}
$$

Consider now the case $\rho<0$. We can write (4.3) in a different way by reversing the order of integration as

$$
L(h, k, \rho)=\frac{1}{2 \pi \sqrt{1-\rho^{2}}} \int_{y_{1}=\frac{h+k}{\sqrt{2}}}^{\infty} \int_{y_{2}=-y_{1}+\sqrt{2} h}^{y_{2}=y_{1}-\sqrt{2} k} \exp \left\{-\frac{y_{1}^{2}}{2(1+\rho)}-\frac{y_{2}^{2}}{2(1-\rho)}\right\} d y_{2} d y_{1} .
$$

By scaling the variables using (4.4), we get

$$
\begin{aligned}
L(h, k, \rho)= & \frac{1}{\pi}\left\{\int_{z_{1}=z_{10}}^{\infty} \int_{z_{2}=0}^{z_{2}=a_{2} z_{1}+b_{2}} e^{-z_{1}^{2}-z_{2}^{2}} d z_{2} d z_{1}\right. \\
& \left.+\int_{z_{1}=z_{10}}^{\infty} \int_{z_{2}=-a_{2} z_{1}-c_{2}}^{\infty} e^{-z_{1}^{2}-z_{2}^{2}} d z_{2} d z_{1}\right\} \\
= & \frac{1}{\pi}\left\{\int_{z_{1}=z_{10}}^{\infty} \int_{z_{2}=0}^{a_{2} z_{1}+b_{2}} e^{-z_{1}^{2}-z_{2}^{2}} d z_{2} d z_{1}+\int_{z_{1}=z_{10}}^{\infty} \int_{z_{2}=0}^{z_{2}=a_{2} z_{1}+c_{2}} e^{-z_{1}^{2}-z_{2}^{2}} d z_{2} d z_{1}\right\},
\end{aligned}
$$

where

$$
a_{2}=\sqrt{\frac{1+\rho}{1-\rho}}=\frac{1}{a_{1}}, b_{2}=\frac{-k}{\sqrt{1-\rho}}, c_{2}=\frac{-h}{\sqrt{1-\rho}}, z_{10}=\frac{h+k}{2 \sqrt{1+\rho}} .
$$

Following the same procedure, we get for $\rho<0$,

$$
\begin{gathered}
L(h, k, \rho)=L_{20}+\sum_{u=0}^{\infty}\left[C_{u}\left(1-P\left(u+1, z_{10}^{2}\right)\right)\left(e^{-b_{2}^{2}} H_{2 u}\left(b_{2}\right)+e^{-c_{2}^{2}} H_{2 u}\left(c_{2}\right)\right)\right. \\
\left.-D_{u}\left(1-\operatorname{sign}\left(z_{10}\right) P\left(u+\frac{3}{2}, z_{10}^{2}\right)\right)\left(e^{-b_{2}^{2}} H_{2 u+1}\left(b_{2}\right)+e^{-c_{2}^{2}} H_{2 u+1}\left(c_{2}\right)\right)\right]
\end{gathered}
$$

where

$$
\begin{gathered}
C_{u}=\frac{1}{2 \sqrt{\pi} \Gamma\left(u+\frac{3}{2}\right)}\left(\frac{a_{2}}{2}\right)^{2 u+1}, D_{u}=\frac{1}{2 \sqrt{\pi} \Gamma(u+2)}\left(\frac{a_{2}}{2}\right)^{2 u+2}, \\
L_{20}=\frac{1}{2}\left(1-\operatorname{sign}\left(z_{10}\right)\left(2 \Phi\left(\sqrt{2}\left|z_{10}\right|\right)-1\right)\right)\left(1-\Phi\left(-\sqrt{2} b_{2}\right)-\Phi\left(-\sqrt{2} c_{2}\right)\right) .
\end{gathered}
$$

For practical implementation, the following recurrence formulas and initial values can be used:

$$
\begin{gathered}
H_{u+1}(x)=2 x H_{u}(x)-2 u H_{u-1}(x), \\
H_{0}(x)=1, \quad H_{1}(x)=2 x, \\
P\left(u+1, x^{2}\right)=P\left(u, x^{2}\right)-\frac{x^{2 u} e^{-x^{2}}}{\Gamma(u+1)}, \quad \Gamma(u+1)=u \Gamma(u), \\
P\left(1, x^{2}\right)=1-e^{-x^{2}} \quad, \quad P\left(\frac{3}{2}, x^{2}\right)=2 \Phi(\sqrt{2}|x|)-\frac{2|x|}{\sqrt{\pi}} e^{-x^{2}}-1 .
\end{gathered}
$$


One can observe that (4.6) can be written in a more compact form as

$$
\begin{aligned}
L(h, k, \rho)= & L_{20}+\sum_{u=0}^{\infty}\left[\psi_{u}\left(b_{2}\right)+\psi_{u}\left(c_{2}\right)\right] \\
& \cdot\left\{\begin{aligned}
(-1)^{u}\left[1-P\left(\frac{u}{2}+1, z_{10}^{2}\right)\right] & \text { if } h+k \geq 0, \\
{\left[(-1)^{u}-P\left(\frac{u}{2}+1, z_{10}^{2}\right)\right] } & \text { if } h+k<0
\end{aligned}\right.
\end{aligned}
$$

where

$$
\psi_{u}(x)=\frac{1}{2 \sqrt{\pi} \Gamma\left(\frac{u}{2}+\frac{3}{2}\right)}\left(\frac{a_{2}}{2}\right)^{u+1} e^{-x^{2}} H_{u}(x) .
$$

Note that for $\rho>0$, we can evaluate $L(h,-k,-\rho)$ using (4.6) and then use (4.2) to obtain the required integral. Practically using (4.5) directly or (4.6) in conjunction with (4.2) somehow led to similar accuracy and number of terms. So for simplicity, we used the second approach in our numerical experiments. On the other hand, although (4.7) is a more compact form than (4.6), its recurrence formulas are more complicated and thus it is not preferred for practical implementation.

For the special cases $h=k=0$ and $\rho<0$, by using (4.6), we get

$$
\begin{aligned}
L(0,0, \rho) & =\frac{1}{\sqrt{\pi}} \sum_{u=0}^{\infty} \frac{(-1)^{u}}{\Gamma\left(u+\frac{3}{2}\right)}\left(\frac{a_{2}}{2}\right)^{2 u+1} H_{2 u}(0) \\
& =\frac{1}{\pi} \sum_{u=0}^{\infty} \frac{(-1)^{u}}{(2 u+1)}\left(\sqrt{\frac{1+\rho}{1-\rho}}\right)^{2 u+1}=\frac{1}{\pi} \arctan \left(\sqrt{\frac{1+\rho}{1-\rho}}\right) \\
& =\frac{1}{2 \pi} \arccos (-\rho)=\frac{1}{2 \pi}\left[\frac{\pi}{2}+\arcsin \rho\right]=\frac{1}{4}+\frac{1}{2 \pi} \arcsin \rho \text { for } \rho<0,
\end{aligned}
$$

where we have used the following formulas (Andrews, 1985, p. 173):

$$
H_{2 u}(0)=(-1)^{u} \frac{2 u !}{u !}, \quad H_{2 u+1}(0)=0 .
$$

Similarly, for $h=k=0$ and $\rho>0$, by using (4.6) and (4.2), we get

$$
\begin{aligned}
L(0,0, \rho) & =\frac{1}{2}-\left(\frac{1}{4}-\frac{1}{2 \pi} \arcsin \rho\right) \\
& =\frac{1}{4}+\frac{1}{2 \pi} \arcsin \rho \text { for } \rho>0 .
\end{aligned}
$$

One can see that these two formulas agree with the well-known formula (Johnson and Kotz, 1972)

$$
L(0,0, \rho)=\frac{1}{4}+\frac{1}{2 \pi} \arcsin \rho \text { for all } \rho .
$$

4.3. Error analysis. If we use a partial sum of $N$ terms of (4.6) and using the fact that $0 \leq P\left(x, z_{10}^{2}\right) \leq 1$, the absolute error, $|E|$, is bounded by

$$
|E| \leq \varepsilon_{1}\left(b_{2}\right)+\varepsilon_{1}\left(c_{2}\right)+\theta\left[\varepsilon_{2}\left(b_{2}\right)+\varepsilon_{2}\left(c_{2}\right)\right],
$$


where

$$
\begin{gathered}
\varepsilon_{1}(x)=\sum_{u=N}^{\infty}\left|\frac{1}{2 \sqrt{\pi} \Gamma\left(u+\frac{3}{2}\right)}\left(\frac{a_{2}}{2}\right)^{2 u+1} e^{-x^{2}} H_{2 u}(x)\right|, \\
\varepsilon_{2}(x)=\sum_{u=N}^{\infty}\left|\frac{1}{2 \sqrt{\pi} \Gamma(u+2)}\left(\frac{a_{2}}{2}\right)^{2 u+2} e^{-x^{2}} H_{2 u+1}(x)\right|, \\
\theta= \begin{cases}1 & \text { if } h+k \geq 0, \\
2 & \text { if } h+k<0 .\end{cases}
\end{gathered}
$$

Using the substitution, $p=u-N$, we get

$$
\varepsilon_{1}(x)=\sum_{p=0}^{\infty}\left|\frac{1}{2 \sqrt{\pi} \Gamma\left(N+p+\frac{3}{2}\right)}\left(\frac{a_{2}}{2}\right)^{2 N+2 p+1} e^{-x^{2}} H_{2 N+2 p}(x)\right| .
$$

Using (3.1), we get:

$$
\begin{aligned}
\varepsilon_{1}(x) & \leq \sum_{p=0}^{\infty} \frac{2^{2 N+2 p}(N+p) !}{2 \sqrt{\pi} \Gamma\left(N+p+\frac{3}{2}\right)}\left(\frac{a_{2}}{2}\right)^{2 N+2 p+1} \exp \left(-\frac{x^{2}}{2}\right)\left[2-\frac{(2 N+2 p) !}{2^{2 N+2 p}(N+p) !^{2}}\right] \\
& \leq \frac{1}{2 \sqrt{\pi}} \exp \left(-\frac{x^{2}}{2}\right) \sum_{p=0}^{\infty} a_{2}^{2 N+2 p+1}=\frac{a_{2}^{2 N+1} \exp \left(-\frac{x^{2}}{2}\right)}{2 \sqrt{\pi}\left(1-a_{2}^{2}\right)}
\end{aligned}
$$

Similarly, $\varepsilon_{2}(x)$ can be bounded using (3.2) as

$$
\begin{aligned}
\varepsilon_{2}(x) & \leq \sum_{p=0}^{\infty} \frac{1}{2 \sqrt{\pi} \Gamma(N+p+2)}\left(\frac{a_{2}}{2}\right)^{2 N+2 p+2} e^{-x^{2}}\left|H_{2 N+2 p+1}(x)\right| \\
& \leq \sum_{p=0}^{\infty} \frac{|x| \exp \left(-\frac{x^{2}}{2}\right)}{2 \sqrt{\pi} \Gamma(N+p+2)}\left(\frac{a_{2}}{2}\right)^{2 N+2 p+2}\left[\frac{\Gamma(2 N+2 p+3)}{\Gamma(N+p+2)}\right] \\
& \leq \frac{|x| \exp \left(-\frac{x^{2}}{2}\right)}{2 \pi} \sum_{p=0}^{\infty} a_{2}^{2 N+2 p+2}=\frac{|x| \exp \left(-\frac{x^{2}}{2}\right) a_{2}^{2 N+2}}{2 \pi\left(1-a_{2}^{2}\right)} .
\end{aligned}
$$

Therefore, the upper bound for the maximum error is given by

$$
|E| \leq \frac{a_{2}^{2 N+1}}{2 \sqrt{\pi}\left(1-a_{2}^{2}\right)}\left\{\exp \left(-\frac{b_{2}^{2}}{2}\right)\left(1+\frac{\theta a_{2}\left|b_{2}\right|}{\sqrt{\pi}}\right)+\exp \left(-\frac{c_{2}^{2}}{2}\right)\left(1+\frac{\theta a_{2}\left|c_{2}\right|}{\sqrt{\pi}}\right)\right\} .
$$

This can be used efficiently to determine in advance the required number of terms to be added for specific values of $\rho, h, k$ to achieve a certain accuracy. For example, for $\rho=-0.95, h=-1, k=-2$, it is adequate to sum the first 9 terms of the series to guarantee that an accuracy of $10^{-15}$ is achieved.

If we follow the same procedure for the tetrachoric series, then a partial sum of $2 N$ terms leads to an absolute error bounded by

$$
|E| \leq \frac{|\rho|^{2 N+1} e^{-\left(h^{2}+k^{2}\right) / 4}}{2 \sqrt{\pi}\left(1-\rho^{2}\right)}\left\{1+\frac{|\rho h k|}{\pi}\right\} .
$$

For the alternative series, if a partial sum of $N$ terms is used, the absolute error, $|E|$, is bounded by (Vasicek, 1998):

$$
|E| \leq \frac{1}{4 \pi(N+0.5)}\left\{\frac{\left(1-\rho_{1}^{2}\right)^{N+0.5}}{\rho_{1}^{2}}+\frac{\left(1-\rho_{2}^{2}\right)^{N+0.5}}{\rho_{2}^{2}}\right\},
$$


where

$$
\rho_{1}=\frac{\rho h-k}{\sqrt{h^{2}-2 \rho h k+k^{2}}} \operatorname{sign}(h), \quad \rho_{2}=\frac{\rho k-h}{\sqrt{h^{2}-2 \rho h k+k^{2}}} \operatorname{sign}(k) .
$$

For Owen's series, since it is composed of two alternating decreasing series and the general term of each approaches 0 at $\infty$, then if a partial sum of $N$ terms is used, the absolute error, $|E|$, is less than the absolute value of the first neglected term, i.e.,

$$
|E| \leq \frac{1}{2 \pi(2 N+1)}\left(a^{2 N+1} P\left(N+1, \frac{h^{2}}{2}\right)+b^{2 N+1} P\left(N+1, \frac{k^{2}}{2}\right)\right),
$$

where

$$
a=\min \left(\left|\frac{k-\rho h}{h \sqrt{1-\rho^{2}}}\right|,\left|\frac{h \sqrt{1-\rho^{2}}}{k-\rho h}\right|\right), \quad b=\min \left(\left|\frac{h-\rho k}{k \sqrt{1-\rho^{2}}}\right|,\left|\frac{k \sqrt{1-\rho^{2}}}{h-\rho k}\right|\right) .
$$

4.4. Numerical experiments. We compared different series expansions for the bivariate normal complementary integral. Methods compared are: Pearson method (tetrachoric), Vasicek method (alternative) and our proposed method (novel). Since the formulation of Vasicek is valid only for $h k \neq 0$, for fair comparison, values examined for $\rho$ are $\{-0.99,-0.90,-0.80, \ldots,-0.10,-0.01,0.01,0.10,0.20, \ldots, 0.90,0.99\}$ and those for both $h$ and $k$ are $\{-4.0,-3.9, \ldots-0.1,-0.01,0.01,0.1,0.2, \ldots, 4\}$. We selected the Owen-Donnelly algorithm (Donnelly, 1973) as our benchmark since it has been considered the most widely implemented with 15 significant digits accuracy. We also compared the results with the Gauss quadrature method implemented by Genz (2004) (see: www.math.wsu.edu/faculty/genz/software/software.html). For each series, we examined the partial sums until an accuracy of $10^{-15}$ was obtained or the maximum number of terms (200 in our experiments) was reached. The results are reported in Table 1 where 10 runs are established and the average total time $(T)$ required to evaluate the integral for each $\rho$ over the experimental range of $h$ and $k$ is reported and its standard deviation (between brackets). The absolute error is defined as the absolute difference between $L(h, k, \rho)$ obtained by the Owen-Donnelly algorithm and the algorithms compared and its maximum over the experimental range of $h$ and $k$ is denoted by $\left|E_{\text {Owen }}\right|$. The maximum number of terms added for each $\rho$ and over the experimental range of $h$ and $k$ is denoted by $N_{\max }$. We used MATLAB 7.1 on Windows XP with SP2 running on Pentium 2.4GHz PC with 3GB RAM. It can be observed that the tetrachoric series converges fast (see $T$ and $N$ in the table) for small $|\rho|$ as expected, however, it converges slowly as $|\rho|$ is close to 1 . On the other hand, the novel series converges fast when $|\rho|$ is close to 1 and slowly as $|\rho|$ approaches 0 . For the alternative series, it converges fast for large $|\rho|$, however, its rate of convergence is very low when $\rho h$ is close to $k$ or $\rho k$ is close to $h$. These situations result in a maximum absolute error for the alternative series much higher compared to both the tetrachoric and the novel series even if we added the maximum number of terms (200). It can also be noted that the Gauss quadrature method Genz (2004) achieves the required accuracy in a reasonable time for the whole range of $\rho$, but overall it is slower than the novel series for large $|\rho|$ and the tetrachoric series for small $|\rho|$. In summary, the fastest of the competing methods (for obtaining an accuracy of $10^{-15}$ ) are the tetrachoric series for $|\rho|<0.5$ and the novel series when $|\rho| \geq 0.5$. 


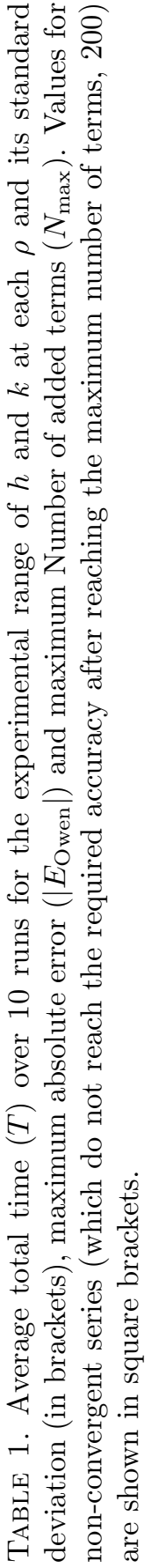

\begin{tabular}{|c|c|c|}
\hline & & 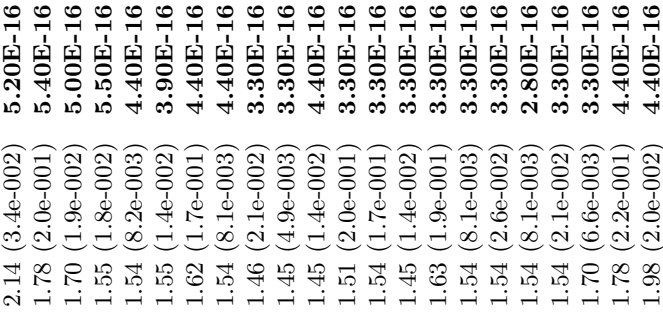 \\
\hline & & 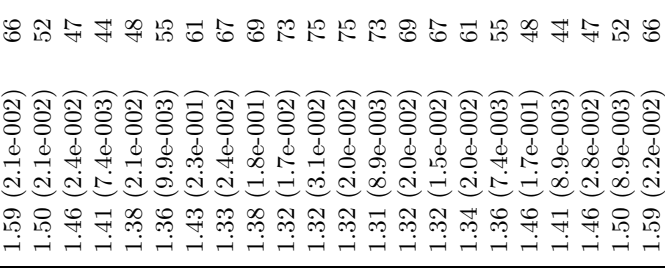 \\
\hline & & 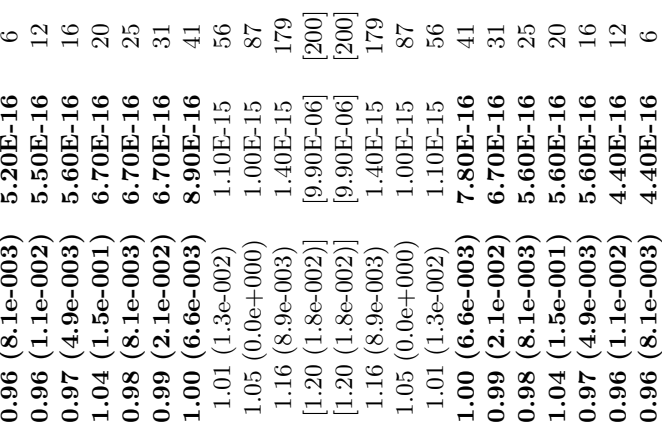 \\
\hline & & 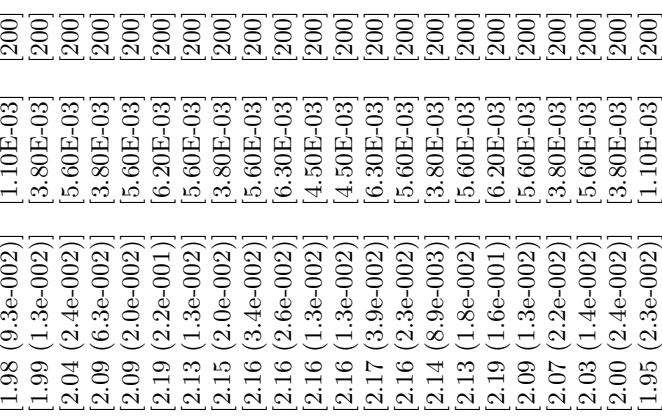 \\
\hline & & 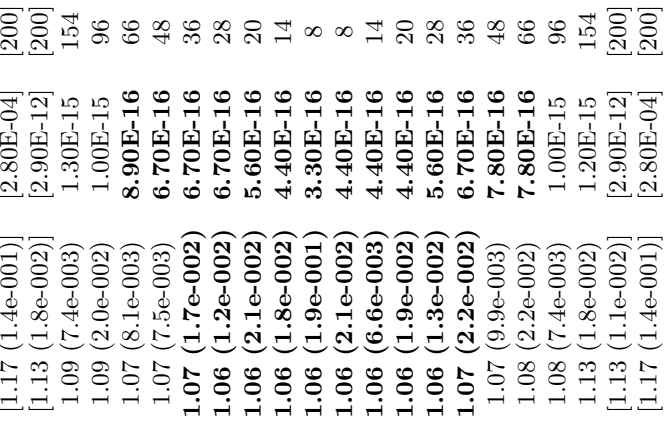 \\
\hline & & \\
\hline
\end{tabular}




\section{Conclusions}

In this paper, we expressed the integral of the product of the error function and the normal probability density as an infinite series of the Hermite polynomial and the normalized incomplete Gamma function. Then we showed that this expression can be used effectively to derive novel series expansions for the bivariate normal integral. These series converge fast for large $|\rho|$ and hence require partial sums of a small number of terms, compared to the tetrachoric series. According to our numerical results, we recommend using the tetrachoric series for $|\rho|<0.5$ and our novel series when $|\rho| \geq 0.5$ to achieve good accuracy fast using a small number of terms.

\section{REFERENCES}

M. Abramowitz, I.A. Stegun, Handbook of mathematical functions, Dover, New York, 1964.

L.C. Andrews, Special functions for engineers and applied mathematicians, MacMillan, New York, 1985. MR779819 (86g:33001)

T.M. Apostol. Lerch's transcendent, in: F.W.J. Olver, D.W. Lozier,R.F. Boisvert, C.W. Clark (Eds), NIST Handbook of mathematical functions, Cambridge University Press, 601-615, 2010. MR2723248 (2012a:33001)

K. Briggs, http://keithbriggs.info/documents/erf-integrals.pdf, 2003.

J.W. Dash, Quantitative finance and risk management: a physicist's approach, World Scientific Publishing, River Edge, New Jersey, 2004. MR2090677|(2005h:91001)

D.R. Divgi, Calculation of univariate and bivariate normal probability functions, Ann. Stat. 7 (1979), 903-910. MR532253 (80d:62021)

T.G. Donnelly, Algorithm 462: Bivariate normal distribution, Commun. ACM 16 (1973), 636.

Z. Drezner, Computation of the bivariate normal integral, Math. Comput. 32 (1978), 277-279. MR0461849 (57:1833)

Z. Drezner, G.O. Wesolowsky, The computation of the bivariate normal integral, J. Stat. Comput. Simul. 35 (1990), 101-107. MR1041725

W.N. Evans, R.M. Schwab, Finishing high school and starting college: do catholic schools make a difference?, J. Econ. 110 (4) (1995), 941-974.

J. Gai, A computational study of the bivariate normal probability function, M.Sc. thesis, Department of Mathematics and Statistics, Queen's University, Kingston, Ontario, Canada, 2002.

A. Genz, Numerical computation of rectangular bivariate and trivariate normal and $\mathrm{t}$ probabilities, Stat. Comput. 14 (3) (2004), 251-260. MR2086401

N.L. Johnson, S. Kotz, Distributions in statistics: Continuous multivariate distribution, John Wiley and Sons, New York, 1972. MR0418337

S.H. Martzoukos, The option on $n$ assets with exchange rate and exercise price risk. J. Multinatl. Financ. Manage. 11 (1) (2001), 1-15.

C. Nicholson, The Probability Integral for Two Variables, Biometrika 33 (1) (1943), 59-72. MR0011409 (6:161f)

D.B. Owen, Tables for computing bivariate normal probabilities, Ann. Math. Stat. 27 (1956), 1075-1090. MR0127562 (23:B607)

K. Pearson, Mathematical contributions to the theory of evolution. VII. on the correlation of characters not quantitatively. Philos. Trans. R. Soc. S-A. 196 (1901), 1-47.

K. Pearson, Mathematical contributions to the theory of evolution. XI. on the influence of natural selection on the variability and correlation of organs. Philos. Trans. R. Soc. S-A. 200 (1903), 1-66. 
W.F. Sheppard, On the calculation of the double-integral expressing normal correlation, Trans. Camb. Philos. Soc. 19 (1900), 23-66.

M.K. Simon, D. Divsalar, Some new twists to problems involving the Gaussian probability integral, IEEE Trans. Commun. 46 (2) (1998), 200-210.

J. Terza, U. Welland, A comparison of bivariate normal algorithms, J. Stat. Comput. Simul. 39 (1991), 115-127.

O.F. Vasicek, A series expansion for the bivariate normal integral, J. Comput. Financ. 1 (1998), 5-10.

J.M. Wooldridge, Econometric analysis of cross section and panel data. MIT Press, Cambridge, MA, 2001. MR2768559

Department of Engineering Mathematics and Physics, Faculty of Engineering, Cairo University, Cairo, Egypt 12613

E-mail address: $\mathrm{h}_{-}$fayed@eng.cu.edu.eg

Department of Computer Engineering, Faculty of Engineering, Cairo University, CAIRo, Egypt 12613

E-mail address: amir@alumni.caltech.edu 